\title{
Immunosuppressive cell death in cancer
}

\author{
Lorenzo Galluzzi, Aitziber Buqué, Oliver Kepp, Laurence Zitvogel \\ and Guido Kroemer
}

In our recent Review (Immunogenic cell death in cancer and infectious disease. Nat. Rev. Immunol. 17, 97-111 (2017)) $)^{1}$, we discussed the molecular and cellular processes through which the death of infected and malignant cells can initiate an adaptive immune response against dead cellassociated antigens (in immunocompetent syngeneic hosts). This process, which is commonly known as immunogenic cell death (ICD), involves: first, the timely release of danger signals from dying cells secondary to the activation of adaptive stress responses, which include the unfolded protein response ${ }^{2}$ and autophagy ${ }^{3}$; and second, the proficient detection of such signals by innate and adaptive compartments of the immune system ${ }^{4}$. Preclinical and clinical data support the notion that ICD has prominent pathophysiological implications in the context of cancer therapy ${ }^{1}$. Indeed, molecular defects in the mechanisms that underlie the release or detection of ICD-associated danger signals have been associated with poor disease outcome in multiple cohorts of patients with cancer ${ }^{5-7}$.

In their Correspondence (Immunosuppressive cell death in cancer. Nat. Rev. Immunol. doi: 10.1038/nri.2017.46 (2017)), Xia and collaborators correctly point out that the demise of malignant cells responding to chemotherapy or radiation therapy frequently fails to elicit anticancer immunity in preclinical and clinical scenarios. Indeed, far too often neoplasms progress as they harness strategies to evade or suppress potential ICDdriven immune responses ${ }^{8-10}$. Furthermore, chemotherapy and radiation therapy are often administered to patients at high doses, which have immunosuppressive rather than immunostimulatory effects ${ }^{11-13}$. Thus, cancer cell death - as elicited in patients by conventional chemotherapeutic and radiotherapeutic regimens - is intrinsically prone to be overlooked by the immune system or to stimulate immune tolerance. Based on this postulate, $\mathrm{Xia}$ and collaborators propose that combinatorial antineoplastic strategies should be aimed at maximizing the number of cancer cells that succumb to treatment.

We believe that - as long as anticancer therapies are unable to eradicate $100 \%$ of malignant cells (which is obviously a utopian goal) - our efforts should instead concentrate on the development of combinatorial therapeutic regimens that render cancer cell death immunogenic and revert immune exhaustion or suppression. Indeed, accumulating preclinical and clinical data support the notion that the way in which cancer cells succumb in response to treatment may be far more important for long-term disease outcome - which is almost always determined by immunosurveillance - than the actual fraction of cells that $\mathrm{die}^{14}$.

Lorenzo Galluzzi is at the Department of Radiation Oncology, Weill Cornell Medical College, New York, New York 10065, USA; and the Université Paris Descartes/Paris V, Sorbonne Paris Cité, 75006 Paris, France.

Aitziber Buqué and Oliver Kepp are at Université Paris Descartes/Paris V, Sorbonne Paris Cité, 75006 Paris, France; at Université Pierre et Marie Curie/Paris VI, 75006 Paris, France; Equipe 11 labellisée Ligue contre le Cancer, Centre de Recherche des Cordeliers, 75006

Paris, France; INSERM, U1138, 75006 Paris, France; and at Metabolomics and Cell Biology Platforms, Gustave Roussy Comprehensive Cancer Institute, 94805 Villejuif, France.

Laurence Zitvogel is at Gustave Roussy Comprehensive Cancer Institute, 94805 Villejuif, France; INSERM, U1015, 94805 Villejuif, France; the Center of Clinical Investigations in Biotherapies of Cancer (CICBT) 1428, 94805 Villejuif, France; and the Universite Paris Sud/ Paris XI, 94270 Le Kremlin-Bicêtre, France.

Guido Kroemer is at Université Paris Descartes/Paris V, Sorbonne Paris Cité, 75006 Paris, France; Université Pierre et Marie Curie/Paris VI, 75006 Paris; Equipe 11 labellisée Ligue contre le Cancer, Centre de Recherche des Cordeliers, 75006 Paris, France; at INSERM, U1138, 75006 Paris, France; Metabolomics and Cell Biology Platforms, Gustave Roussy Comprehensive Cancer Institute, 94805 Villejuif, France; Karolinska Institute, Department of Women's and Children's Health, Karolinska University Hospital, 17176 Stockholm, Sweden; and at Pôle de Biologie, Hopitâl Européen George Pompidou, AP-HP; 75015 Paris, France.

Correspondence to L.G. and G.K. deadoc@vodafone.it; kroemer@orange.fr doi:10.1038/nri.2017.48 Published online 8 May 2017
1. Galluzzi, L., Buque, A., Kepp, O., Zitvogel, L. \& Kroemer, G. Immunogenic cell death in cancer and infectious disease. Nat. Rev. Immunol. 17, 97-111 (2017).

2. Bettigole, S. E. \& Glimcher, L. H. Endoplasmic reticulum stress in immunity. Annu. Rev. Immunol. 33 107-138 (2015)

3. Galluzzi, L., Bravo-San Pedro, J. M., Levine, B., Green, D. R. \& Kroemer, G. Pharmacological modulation of autophagy: therapeutic potential and persisting obstacles. Nat. Rev. Drug Discov. http:// dx.doi.org/10.1038/nrd.2017.22 (2017).

4. Krysko, D. V. et al. Immunogenic cell death and DAMPs in cancer therapy. Nat. Rev. Cancer 12 , 860-875 (2012).

5. Kroemer, G., Galluzzi, L., Kepp, O. \& Zitvogel, L. Immunogenic cell death in cancer therapy. Annu. Rev. Immunol. 31, 51-72 (2013).

6. Fucikova, J. et al. Prognostic and predictive value of DAMPs and DAMP-associated processes in cancer. Front. Immunol. 6, 402 (2015).

7. Fucikova, J. et al. Calreticulin exposure by malignant blasts correlates with robust anticancer immunity and improved clinical outcome in AML patients. Blood 128, 3113-3124 (2016).

8. Bidwell, B. N. et al. Silencing of Irf7 pathways in breast cancer cells promotes bone metastasis through immune escape. Nat. Med. 18, 1224-1231 (2012).

9. Katlinski, K. V. et al. Inactivation of interferon receptor promotes the establishment of immune privileged tumor microenvironment. Cancer Cell 31, 194-207 (2017).

10. Mohme, M., Riethdorf, S. ¿ Pantel, K. Circulating and disseminated tumour cells - mechanisms of immune surveillance and escape. Nat. Rev. Clin. Oncol. 14 155-167 (2017)

11. Dewan, M. Z. et al. Fractionated but not single-dose radiotherapy induces an immune-mediated abscopal effect when combined with anti-CTLA-4 antibody. Clin. Cancer Res. 15, 5379-5388 (2009)

12. Pasquier, E., Kavallaris, M. \& Andre, N. Metronomic chemotherapy: new rationale for new directions. Nat. Rev. Clin. Oncol. 7, 455-465 (2010).

13. Vanpouille-Box, C. et al. DNA exonuclease Trex 1 regulates radiotherapy-induced tumor

immunogenicity. Nat. Commun. (in the press) (2017).

14. Galluzzi, L., Bravo-San Pedro, J. M., Demaria, S., Formenti, S. C. \& Kroemer, G. Activating autophagy to potentiate immunogenic chemotherapy and radiation therapy. Nat. Rev. Clin. Oncol. 14, 247-258 (2017).

\section{Acknowledgements}

L.G. is supported by a start-up intramural grant from the Department of Radiation Oncology of Weill Cornell Medical College, New York, NY, USA, and Sotio a.c., Prague, Czech Replublic. A.B., O.K. and G.K. are supported by the French Ligue contre le Cancer (équipe labellisêe); the French Agence National de la Recherche (ANR) - Projets Blancs; ANR under the frame of E-Rare-2 (the ERA-Net for Research on Rare Diseases); Association pour la recherche sur le cancer (ARC); Cancéropôle Ile-de-France; the French Institut National du Cancer (INCa); Institut Universitaire de France; Fondation pour la Recherche Médicale (FRM); the European Commission (ArtForce); the European Research Council (ERC); the LeDucq Foundation; the LabEx Immuno-Oncology; the Sites de Recherche Intégrée sur le Cancer (SIRIC) Stratified Oncology Cell DNA Repair and Tumor Immune Elimination (SOCRATE); the SIRIC Cancer Research and Personalized Medicine (CARPEM); and the Paris Alliance of Cancer Research Institutes (PACRI).

Competing interests statement

The authors declare no competing interests. 